Published in final edited form as:

Biomacromolecules. 2016 March 14; 17(3): 778-787. doi:10.1021/acs.biomac.5b01511.

\title{
Chimeric Aptamer-Gelatin Hydrogels as an Extracellular Matrix Mimic for Loading Cells and Growth Factors
}

\author{
Xiaolong Zhang, Mark R. Battig, Niancao Chen, Erin R. Gaddes, Katelyn L. Duncan, and \\ Yong Wang \\ Department of Biomedical Engineering, College of Engineering, The Pennsylvania State \\ University, University Park, PA 16802, USA
}

\begin{abstract}
It is important to synthesize materials to recapitulate critical functions of biological systems for a variety of applications such as tissue engineering and regenerative medicine. The purpose of this study was to synthesize a chimeric hydrogel as a promising extracellular matrix (ECM) mimic using gelatin, a nucleic acid aptamer and polyethylene glycol (PEG). This hydrogel had a macroporous structure that was highly permeable for fast molecular transport. Despite its high permeability, it could strongly sequester and sustainably release growth factors with high bioactivity. Notably, growth factors retained in the hydrogel could maintain $~ 50 \%$ bioactivity during a 14-day release test. It also provided cells with effective binding sites, which led to high efficiency of cell loading into the macroporous hydrogel matrix. When cells and growth factors were co-loaded into the chimeric hydrogel, living cells could still be observed by day 14 in a static serum-reduced culture condition. Thus, this chimeric aptamer-gelatin hydrogel constitutes a promising biomolecular ECM mimic for loading cells and growth factors.
\end{abstract}

\section{INTRODUCTION}

The extracellular matrix $(\mathrm{ECM})$ is a multifunctional material comprised of insoluble macromolecules (e.g., proteoglycans), soluble signaling molecules (e.g., growth factors), and adhesion ligands (e.g., fibronectin) ${ }^{1-3}$ Cells communicate with these ECM components to acquire essential biophysical and biochemical cues for the regulation of proliferation, migration, differentiation, apoptosis, or other functions. ${ }^{4-9}$ Thus, biomaterials with the ability to recapitulate the functions of the ECM are promising cell carriers for both basic biological research and potential applications such as regenerative medicine, drug screening, bioreactor design and molecular sensing. ${ }^{10-16}$

Hydrogels have received great attention for the synthesis of biomimetic cell-carrying materials since they contain a large amount of water with structural similarities to the ECM. ${ }^{17}$ Both natural and synthetic polymers such as alginate and polyethylene glycol (PEG) have been extensively studied for the synthesis of hydrogels. ${ }^{18-21}$ Since most of these hydrogels lack the essential components of the ECM, they need to be functionalized with biomolecules to not only provide cells with binding sites for attachment but also sequester soluble signaling molecules for sustained cell stimulation. ${ }^{22-25}$ It is also important to develop hydrogels with a macroporous structure that ensures sufficient space for cell migration and infiltration as well as free transport of nutrients and wastes. ${ }^{26,27}$ However, to 
our knowledge, few reports have shown that a macroporous cell-carrying hydrogel can not only stably sequester soluble signaling molecules for enhanced cell survival and stimulation but also simultaneously permit free molecular transport of nutrients and wastes. Such a hydrogel will be particularly important for in vitro development of large-scale engineered tissue and in vivo implantation of cells for regenerative medicine.

The purpose of this work was to study a macroporous aptamer-gelatin hydrogel with the abilities to permit free molecular transport, to sequester signaling molecules and to display cell binding sites. Oligonucleotide aptamers are an emerging class of synthetic ligands that are selected from DNA/RNA libraries via a combinatorial chemistry technique, i.e., systematic evolution of ligands by exponential enrichment (SELEX) ${ }^{28,29}$ Because of their high binding affinity and specificity, oligonucleotide aptamers have been studied for a variety of biological and biomedical applications ranging from biomedical nanotechnology to biomaterials science. ${ }^{30-39}$ Our recent studies have demonstrated that oligonucleotide aptamers are promising for the functionalization of hydrogels in protein drug delivery. ${ }^{37-39}$ However, the biological functions of those hydrogels have never been validated at the cell level. Moreover, these hydrogels lack the cell binding function and therefore cannot be used as the ECM mimic for carrying cells. Gelatin, a chemically processed protein product derived from collagen with integrin binding sites, has been widely used for the synthesis of hydrogels by itself or with other polymers. ${ }^{40-42}$ It was hypothesized that a chimeric aptamer-gelatin hydrogel would be a promising biomaterial for loading cells and soluble signaling molecules, which has not been explored before.

To test the hypothesis, the chimeric aptamer-gelatin hydrogel was synthesized with free radical polymerization coupled with gas formation to generate a macroporous structure. Vascular endothelial growth factor (VEGF) and human umbilical vein endothelial cells (HUVECs) were used as a model to study the functions of the chimeric hydrogel. The macroporous structure was evaluated with scanning electron microscopy. The function of cell attachment was investigated both on the two-dimensional hydrogel surface and in the three-dimensional macroporous hydrogel. The function of sequestering VEGF for sustained release was systemically investigated in different release environments. Since the bioactivity of growth factors is critical to the function of a hydrogel, the ability of HUVECs to survive and form tubes was examined. Finally, the chimeric hydrogel loaded with VEGF and HUVECs was evaluated at a static, serum-reduced culture condition to examine the potential of the chimeric hydrogel in mimicking the ECM to carry both growth factors and cells.

\section{MATERIALS AND METHODS}

\subsection{Chemical reagents}

Gelatin from bovine skin (type B), methacrylic acid N-hydroxysuccinimide ester (MANHS), polyethylene glycol (PEG) methyl ether acrylate (PEGMA; Mn = 480), poly(ethylene glycol) diacrylate (PEGDA) $(\mathrm{Mn}=700)$ and Pluronic F-127 (PF127) were purchased from Sigma-Aldrich (St. Louis, MO). Dialysis cassettes $(\mathrm{MWCO}=10 \mathrm{kDa})$, centrifugal filters (MWCO $=10 \mathrm{kDa}$ ), coomassie blue G-250 stain solution, Dulbecco's phosphate buffered saline (DPBS), bovine serum albumin (BSA), sodium bicarbonate, glacial acetic acid, ammonium persulfate (APS), and tetramethylethylenediamine (TEMED) were obtained 
from Fisher Scientific (Suwanee, GA). HUVECs, Medium 200 (M200), low serum growth supplement (LSGS), Geltrex, Hoechst 33342, calcein AM, and ethidium homodimer-1 were purchased from Life Technologies (Grand Island, NY). EndoGRO-VEGF complete media kit was purchased from Millipore (Billerica, MA). Vascular endothelial growth factor-165 (VEGF, MW = 38.2 kDa) and VEGF enzyme-linked immunosorbent assay (ELISA) kit were obtained from Peprotech (Rocky Hill, NJ). The CellTiter 96 AQueous One Solution Cell Proliferation Assay (i.e., MTS assay) kits were obtained from Promega (Madison, WI). The anti-VEGF RNA aptamer (5'/Acrydite/mGmAmGmGmA fCmGmAfUmG fCmGmGAA fUfCmAmGfU mGmAmAfUmG fCfUfUmAfU mAfCmAfUfCfCmG $\left.\left(3^{\prime}-3^{\prime} \mathrm{dT}\right) / 5^{\prime}\right)^{43}$ was purchased from TriLink (San Diego, CA); $m$ indicates a 2'O-methyl modification and $\mathrm{f}$ indicates a $2^{\prime}$ fluorine modification. The inactivator of the anti-VEGF aptamer (5'/FAM/GATTC ACTGA TTCCG CATCG TCCTC/3') was purchased from Integrated DNA Technologies (Coralville, IA).

\subsection{Functionalization of gelatin with MA-NHS}

Gelatin was dissolved in $0.1 \mathrm{M}$ sodium bicarbonate buffer to a final concentration of 20 $\mathrm{mg} / \mathrm{mL}$. MA-NHS was dissolved in anhydrous DMSO at a concentration of $200 \mathrm{mg} / \mathrm{mL}$. The MA-NHS solution was added to the gelatin solution at predetermined molar ratios. The solution was incubated at $37^{\circ} \mathrm{C}$ for 1 hour with continuous stirring. The unreacted reagents and byproducts were removed using a dialysis cassette $(\mathrm{MWCO}=10 \mathrm{kDa})$ in distilled water at $37^{\circ} \mathrm{C}$. Following dialysis, the functionalized gelatin (i.e., gelatin-MA) was concentrated using a centrifugal filter (molecular cut-off: $10 \mathrm{kDa}$, Fisher Scientific, Waltham, MA) at $4000 \mathrm{~g}$ for 30 minutes. The gelatin-MA solution was filtered through a $0.22 \mu \mathrm{m}$ filter and stored at $-20^{\circ} \mathrm{C}$ before use.

\subsection{Fluorescamine assay}

The conjugation efficiency of methacrylamide groups onto gelatin was measured according to a fluorescamine method, ${ }^{44}$ in which the fluorescamine dye rapidly reacted with primary amines of protein and formed a complex that can produce fluorescence with a peak emission at $470 \mathrm{~nm}$. Fluorescamine was first dissolved in DMSO at a concentration of $3 \mathrm{mg} / \mathrm{mL}$. Next, $3 \mu \mathrm{L}$ of fluorescamine solution was mixed with $9 \mu \mathrm{L}$ of the gelatin or gelatin-MA solution. The reaction was carried out at room temperature for 15 minutes and the fluorescence intensity was measured using a Nanodrop 3300 fluorospectrometer (Thermo Fisher Scientific, Waltham, MA) at an excitation wavelength of $390 \mathrm{~nm}$ and an emission wavelength of $475 \mathrm{~nm}$. Glycine was used to generate a standard curve showing the relationship between the number of primary amines and the fluorescence intensity.

\subsection{Preparation of the macroporous hydrogel}

Macroporous gelatin-PEG (G-PEG) hydrogels were prepared using free radical polymerization coupled with gas formation. ${ }^{45}$ A pregel solution $(120 \mu \mathrm{L})$ was prepared by mixing $70 \mu \mathrm{L}$ gelatin-MA (15 mg/mL), $8 \mu \mathrm{L}$ PEG methyl ether acrylate (100\%), $6 \mu \mathrm{L}$ PF127 $(10 \%, \mathrm{w} / \mathrm{v}), 1 \mu \mathrm{L}$ TEMED, $3 \mu \mathrm{L}$ acetic acid, $4 \mu \mathrm{L}$ APS $(10 \%$, w/v), various amount of acrydite-aptamer $(2.5 \mathrm{pmol}, 12.5 \mathrm{pmol}, 25 \mathrm{pmol}, 50 \mathrm{pmol})$, and $\mathrm{diH}_{2} \mathrm{O} .10 \mu \mathrm{L}$ of the pregel solution was added into a mold containing $0.05 \mathrm{~g}$ of sodium bicarbonate. The pregel solution reacted with the sodium bicarbonate for the production of $\mathrm{CO}_{2}$ during the formation of the 
hydrogel. The sodium bicarbonate particles were well distributied in the bottom of mold for ensuring a homogeneous $\mathrm{CO}_{2}$ buble formation in hydrogel synthesis. The molarity of gelatin was $175 \mu \mathrm{M}$ in each hydrogel. PEG diacrylate $(15 \%$, v/v) was used to synthesize pure PEG hydrogel in the presence of the free radical and gas forming reagents. The hydrogels were washed with $1 \mathrm{~mL}$ sterilized $\mathrm{diH}_{2} \mathrm{O}$ overnight to remove any unreacted monomers, $\mathrm{PF} 127$, ions, TEMED and APS. The hydrogels were sterilized with 70\% ethanol for 1 hour and subsequently washed three washes with $1 \mathrm{~mL}$ DPBS.

\subsection{Examination of gelatin and oligonucleotide aptamer incorporation into the macroporous hydrogel}

To examine the gelatin incorporation, the macroporous G-PEG hydrogels were incubated with Coomassie Blue G-250 staining solution. After 1 hour incubation, the macroporous hydrogels were washed in the destaining solution ( $40 \%$ methanol, $10 \%$ glacial acetic acid, and $50 \% \mathrm{diH}_{2} \mathrm{O}$ ) at $37^{\circ} \mathrm{C}$ for 2 hours with the destaining solution replenished every 30 minutes. The macroporous hydrogels were imaged under a Maestro Imaging System (PerkinElmer, Waltham, MA). PEG diacrylate (15\%, v/v) was used for synthesizing a gelatin-free hydrogel as a control. To examine the aptamer incorporation, the macroporous hydrogels (with 50 pmol of aptamer) were incubated overnight with 200 pmol of a FAMlabeled complementary oligonucleotide that binds to the anti-VEGF aptamer. The hydrogels were imaged with the Maestro Imaging System (PerkinElmer, Waltham, MA) after thorough washing with the DPBS buffer to remove free complementary oligonucleotides.

\subsection{Preparation of the G-PEG hydrogel film on the glass for cell adhesion}

A pregel solution $(12 \mu \mathrm{L})$ was prepared by combining $7 \mu \mathrm{L}$ gelatin-MA $(15 \mathrm{mg} / \mathrm{mL}), 0.6 \mu \mathrm{L}$ PF127 (10\%, w/v), $0.1 \mu \mathrm{L}$ TEMED, $0.3 \mu \mathrm{L}$ acetic acid, $0.4 \mu \mathrm{L}$ APS (10\%, w/v), various amount of PEG methyl ether acrylate and $\mathrm{diH}_{2} \mathrm{O} .1 \mu \mathrm{L}$ of pregel solution was added onto a silanized glass substrate $(4 \mathrm{~mm} \times 4 \mathrm{~mm})$, which was then covered by a cover slide. ${ }^{46}$ After 30 minutes, the cover slide was gently removed. The molarity of gelatin was $175 \mu \mathrm{M}$ in each hydrogel. PEG diacrylate (15\%, v/v) was used for synthesizing a pure PEG hydrogel film as a control. The hydrogels were washed with $1 \mathrm{~mL}$ sterilized $\mathrm{diH}_{2} \mathrm{O}$ overnight to remove any unreacted monomers, PF127, ions, TEMED and APS. The hydrogels were sterilized with $70 \%$ ethanol for 1 hour and subsequently washed three times with $1 \mathrm{~mL}$ DPBS.

\subsection{Examination of cell adhesion on the G-PEG hydrogel surface}

HUVECs were harvested and suspended in LSGS-supplemented M200 medium at a concentration of $1.0 \times 10^{5}$ cells $/ \mathrm{mL} .250 \mu \mathrm{L}$ of the HUVECs suspension was added onto the hydrogel films coated on the glass slides and incubated at $37^{\circ} \mathrm{C}$ overnight. Prior to cell imaging, calcein AM and Hoechst 33342 were incubated with the hydrogels for 30 minutes at a concentration of $2 \mu \mathrm{g} / \mathrm{mL}$ each. The hydrogels were washed once using LSGSsupplemented M200 medium before imaging under a fluorescence microscope (Olympus IX73, Pittsburgh, PA). The number of cells on each hydrogel was quantified using the Image J software. 


\subsection{Examination of cell viability}

The viability of attached cells on the hydrogel was measured using a MTS assay. Briefly, hydrogels were transferred into solutions containing $160 \mu \mathrm{L}$ of LSGS-supplemented M200 medium and $40 \mu \mathrm{L}$ of MTS solution in a 96-well plate. After 3 hours of incubation at $37^{\circ} \mathrm{C}$, $100 \mu \mathrm{L}$ of solutions were collected and transferred into a new 96-well plate for absorbance measurement at $490 \mathrm{~nm}$ using an Infinite 200 Pro microplate reader (Tecan, Morrisville, $\mathrm{NC})$.

\subsection{Morphological examination of the macroporous hydrogels}

Hydrogels were lyophilized for one day at $-60{ }^{\circ} \mathrm{C}$. The hydrogels were then mounted on an aluminum stub using double-sided adhesive tape and sputter-coated with a thin layer of gold for 30 seconds. The coated hydrogels were imaged under an environmental scanning electron microscope (FEI Quanta 200, Hillsboro, OR) in high vacuum mode with an accelerating voltage of $15 \mathrm{kV}$.

\subsection{Cell loading into the macroporous hydrogels}

Macroporous hydrogels were dehydrated by gently blotting the hydrogels with sterilized tissue paper. The hydrogels were placed into 96-well plates and $250 \mu \mathrm{L}$ of HUVECs suspensions containing $2.5 \times 10^{4}$ cells (low density), $1.25 \times 10^{5}$ cells (intermediate density), and $6.25 \times 10^{5}$ cells (high density) were slowly added onto the dehydrated macroporous hydrogels. After 1 hour incubation, $1 \mathrm{~mL}$ of fresh LSGS-supplemented M200 medium was added to each hydrogel, followed by overnight incubation. Cells were incubated in $2 \mu \mathrm{g} / \mathrm{mL}$ calcein AM for 30 minutes and then washed once with LSGS-supplemented M200 medium. To image the cells, the hydrogels were cut into half horizontally and transferred into a chamber slide. The cross-sections of the hydrogels were observed using a scanning laser confocal microscope (Olympus FV1000, Centre Valley, PA) equipped with an argon laser. A series of images were collected throughout the z-direction and a three-dimensional composite image was created and presented.

\subsection{Examination of VEGF sequestration}

Macroporous hydrogels were prepared as previously stated. Prior to loading VEGF into the macroporous hydrogels, the hydrogels were dehydrated by gently blotting the hydrogels with sterilized tissue paper. $50 \mu \mathrm{L}$ of the VEGF solution (200 ng and $0.1 \%$ BSA) was added to each hydrogel and the samples were incubated at $4{ }^{\circ} \mathrm{C}$ for 24 hours. After incubation, the VEGF-loaded hydrogel was incubated in $1 \mathrm{~mL}$ of DPBS with $0.1 \%$ BSA for 24 hours. The amount of VEGF in DPBS was measured by ELISA. The sequestration efficiency was determined as the amount of VEGF remaining in the hydrogel over the amount of VEGF loaded into the hydrogel.

\subsection{Examination of the VEGF release kinetics}

The VEGF-loaded macroporous hydrogels with a 5:1 molecular ratio of aptamer to VEGF were incubated in $1 \mathrm{~mL}$ of release medium at $37^{\circ} \mathrm{C}$ with a shaking rate of $70 \mathrm{rpm}$. The VEGF loading amount was $200 \mathrm{ng}$. Three release media were used and compared, including DPBS, M200 and LSGS-supplemented M200. The release media were collected every day 
and replaced with $1 \mathrm{~mL}$ of fresh release medium. The collected release media were stored at $-20^{\circ} \mathrm{C}$ until measurement with ELISA.

\subsection{Tube formation assay}

Two experiments were designed for the tube formation assay. The first experiment was used to compare the controlled release function of the macroporous hydrogels with and without the aptamer. In this experiment, the VEGF-loaded macroporous hydrogels were incubated in $1 \mathrm{~mL}$ of M200 medium at $37^{\circ} \mathrm{C}$ with a shaking rate of $70 \mathrm{rpm}$. The M200 medium was changed every day. The release media collected at day 7 and day 14 were directly used without any treatment for the tube formation assay. The second experiment was used to measure the bioactivity of VEGF remaining in the hydrogels. In this experiment, fresh VEGF was extracted from the hydrogels that were triggered with the complementary sequence of the anti-VEGF aptamer for 1 hour at day 7 and day 14. The collected VEGF solutions were diluted with M200 medium to prepare two solutions containing VEGF of 10 and $20 \mathrm{ng} / \mathrm{mL}$ for the tube formation assay. The VEGF stock solution of the same concentration was used as a positive control.

The tube formation assay was performed according to the standard procedure. ${ }^{47}$ Briefly, the Geltrex solution was first thawed at $4^{\circ} \mathrm{C}$ overnight and $80 \mu \mathrm{L}$ of the Geltrex solution was added into each well of a 48 -well plate for 30 minutes incubation at $37{ }^{\circ} \mathrm{C} .200 \mu \mathrm{L}$ of release medium or VEGF solution were transferred onto the Geltrex-coated wells with $4.0 \times 10^{4}$ HUVECs. After incubation at $37^{\circ} \mathrm{C}$ in $5 \% \mathrm{CO}_{2}$ for 4 hours, the cells were stained with calcein AM $(2 \mu \mathrm{g} / \mathrm{mL})$ for 30 minutes and then observed under a fluorescence microscope (Olympus IX73, Pittsburgh, PA). Cell participation and the total tube length were measured using Image J software. P-value less than 0.05 was considered to be significant. The elongated cells were counted as cells comprising the tube-like structure whereas the roundshaped ones were not.

\subsection{Evaluation of cell viability in the macroporous hydrogels}

A solution $(50 \mu \mathrm{L})$ containing $200 \mathrm{ng}$ VEGF and $6.25 \times 10^{5}$ cells was loaded into dehydrated hydrogels. The hydrogels were incubated at $37^{\circ} \mathrm{C}$ in a $5 \% \mathrm{CO}_{2}$ environment for 1 hour and then incubated in $1 \mathrm{~mL}$ of cell culture medium, which was EndoGRO basal medium supplemented with ascorbic acid $(50 \mu \mathrm{g} / \mathrm{mL})$, hydrocortisone hemisuccinate $(1 \mu \mathrm{g} /$ $\mathrm{mL})$, heparin sulfate $(0.75 \mathrm{U} / \mathrm{mL}), \mathrm{L}$-glutamine $(10 \mathrm{mM})$, and $0.5 \%$ FBS. The cell culture medium was replaced every day. At predetermined time points, the hydrogels were stained with calcein AM and ethidium homodimer-1 (both at $2 \mu \mathrm{g} / \mathrm{mL}$ ) to examine live and dead cells, respectively. The fluorescence intensity of the cells in the hydrogels was evaluated with the signals unmixed under the Maestro Imaging System. The hydrogels were also horizontally cut into halves and examined under the confocal microscope (Olympus FV1000, Center Valley, PA).

\subsection{Examination of the effect of macroporous structures on cell viability}

Hydrogels were loaded with $50 \mu \mathrm{L}$ mixture containing VEGF (200 ng) and HUVECs (6.25 × $10^{5}$ cells). After one hour incubation, the hydrogels were gently dehydrated by blotting the hydrogels with sterilized tissue paper and loaded with $30 \mu \mathrm{L}$ of the Geltrex solution to block 
the macroporous structure of hydrogels. The hydrogels were then incubated in $1 \mathrm{~mL}$ of serum-reduced medium (EndoGRO basal medium supplemented with ascorbic acid (50 $\mu \mathrm{g}$ / $\mathrm{mL})$, hydrocortisone hemisuccinate $(1 \mu \mathrm{g} / \mathrm{mL})$, heparin sulfate $(0.75 \mathrm{U} / \mathrm{mL})$, L-glutamine $(10 \mathrm{mM})$, and $0.5 \% \mathrm{FBS})$. The cell culture medium was replaced every day. At different time points, hydrogels were stained with calcein AM and ethidium homodimer-1 $(2 \mu \mathrm{g} / \mathrm{mL}$ each) for 30 minutes and sectioned horizontally for visualization under a scanning laser confocal microscope (Olympus FV1000, Centre Valley, PA).

\subsection{Statistical analysis}

Differences between two unpaired groups were compared using the Mann-Whitney U test. The Difference was considered to be significant if $\mathrm{P}<0.05$. Data are reported as mean \pm the standard deviation of three samples.

\section{RESULTS AND DISCUSSION}

\subsection{Synthesis of the chimeric hydrogel}

The chimeric hydrogel was synthesized with the three macromonomers including the aptamer, PEG, and gelatin. All of them bore unsaturated double bonds (Supporting Figure 1). PEG and the aptamer were chemically functionalized by the companies with acrylate and methacrylamide groups at their ends, respectively. Gelatin was reacted with MA-NHS to conjugate methacrylamide groups on gelatin (Figure 1A). The number of the methacrylamide groups per gelatin molecule increased with the increasing molar ratio of MA-NHS to gelatin (Figure 1B). When the molar ratio reached 50:1, approximately 20 methacrylate groups were conjugated onto one gelatin molecule.

Since each gelatin-MA had multiple methacrylamide groups, gelatin-MA could be used as a chemical crosslinker for the synthesis of hydrogels via free radical polymerization. To confirm the crosslinking function, gelatin-MA was examined for the synthesis of G-PEG hydrogels with native gelatin as a control. The result shows that PEGMA could form a hydrogel with gelatin-MA but it could not with native gelatin (Figure 1C).

To generate the macroporous hydrogel, a gas forming reaction was coupled with free radical polymerization to produce carbon dioxide bubbles during the formation of the hydrogel (Figure 1D). The pore structure was examined with scanning electron microscopy (SEM). The SEM images showed that pure PEGDA could form a macroporous hydrogel via the coupled reactions (Figure 1E). In contrast, when the same reaction conditions were used, pure gelatin-MA itself could not form a macroporous hydrogel (Figure 1E). This result is different from the data reported in the literature [40] showing that gelatin itself can be used to produce macroporous scaffolds, presumably because different methods were used to generate macroporous structures. Our data also indicates that PEG would promote the formation of macroporous structures through mechanical stabilization when mixed with gelatin-MA. Indeed, the solution of gelatin and PEG could form a macroporous hydrogel via free radical polymerization coupled with gas formation (Figure 1E). In addition to the macroporous structures, we stained gelatin and the aptamer to further validate the chemical incorporation of these molecules into the hydrogel matrix during the reactions. Since Coomassie Blue is able to stain proteins, the staining result showed that gelatin was 
successfully incorporated into the hydrogel (Figure 1F). Moreover, the aptamerfunctionalized hydrogel exhibited strong fluorescence after treated with the FAM-labled sequence complementary of the aptamer, which demonstrates the success of aptamer incorporation (Figure 1G).

\subsection{Examination of cell binding and loading}

The functionality of cell binding was first evaluated on a two-dimensional hydrogel coated on a glass surface. HUVECs were seeded on the surface overnight and then stained with Hoechst 33342 and calcein AM. The fluorescence images show that the cells could not attach to the pure PEG hydrogel (Figure 2A), which is consistent with the literature showing that PEG is resistant to cell binding. ${ }^{48}$ In contrast, the presence of gelatin in the hydrogels led to cell attachment (Figure 2A). The number of the cells on the hydrogel increased from 80 to 524 cells $/ \mathrm{mm}^{2}$ when the molar ratio of PEG to gelatin was changed from 3200:1 to 800:1 (Figure 2B). The data showed that the presence of PEG negatively affected cell adhesion. The level of cell adhesion on the G-PEG hydrogel was increased with the decreased amount of PEG, which is consistent with the results reported in the literature. ${ }^{49}$ The cell imaging analysis was also consistent with the cell viability data (Figure 2C). Thus, the results showed that gelatin in the hydrogel provided the cells with effective binding sites for cell attachment.

Since cells in a tissue interact with the ECM in a three dimensional space, cell loading and cell attachment within the macroporous hydrogel were characterized. Two strategies including one-step and two-step loading methods have been studied for loading cells into polymeric systems. ${ }^{50}$ In the one-step method, cells are mixed with a polymer solution before the formation of the polymeric system. While the overall procedure is simple and straightforward, the synthesis of many polymeric systems often involves harsh conditions. For instance, free radicals produced during free radical polymerization may lead to the decrease or loss of cell bioactivity. ${ }^{51}$ Thus, the two-step method has also been studied for cell loading. In this method, polymeric systems with porous structures are generated, which is followed by cell loading. ${ }^{50}$ The two-step method was used in the current work. The macroporous hydrogel after the synthesis was thoroughly washed to remove free monomers, initiators, catalysts and byproducts and then dehydrated before loaded with HUVECs. After the gentle washing of unbound cells, the hydrogel was cut into two halves to expose the cross-section of the central hydrogel region for confocal microscopy imaging. Consistent with the observation of cell attachment on the two-dimensional PEG hydrogel surface, few cells were observed to stably bind the macroporous PEG hydrogel when the initial cell loading was $6.25 \times 10^{5}$ cells (Figure 2D). In contrast, in the same loading condition, the cell loading efficiency was $76 \%$, i.e., approximately 9,500 cells $/ \mathrm{mm}^{3}$ in G-PEG hydrogel. It was also observed that the cell density in the hydrogel increased with the cell loading amount (Figure 2D). Moreover, the cells could reach the central regions of the macroporous hydrogel with a thickness of $\sim 2.5 \mathrm{~mm}$. Thus, these data show that the macroporous hydrogel with gelatin can provide cells with binding sites for effective cell loading. 


\subsection{Evaluation of VEGF sequestration and release}

After the demonstration of the cell binding and loading function, the macroporous chimeric hydrogel was evaluated to examine the function of VEGF sequestration and release. The aptamer is a chemically modified RNA oligonucleotide with 20 nucleotides modified with $2^{\prime}$-Ome and 15 nucleotides modified with 2'-F (Figure 3A). This aptamer binds VEGF with a Kd value of $10 \mathrm{pM} .{ }^{52}$ We only used one aptamer in this study since our previous results of the relationship between the Kd value and the release kinetics have indicated that an aptamer with this Kd value would be sufficient to prolong the release of loaded growth factors. ${ }^{38}$ The results showed that the Apt (+) hydrogel could effectively sequester VEGF (Figure 3B). The sequestration efficiency increased with the density of the aptamer, reaching $91.6 \%$ at the density of $2.5 \mathrm{nmol} / \mathrm{cm}^{3}$ (Figure 3B). In contrast, Apt (-) hydrogel had a poor capability of sequestering soluble signaling molecules, with a VEGF sequestration efficiency of only 5\% (Figure 3B). The poor VEGF retention in Apt (-) hydrogel, on the other hand, demonstrates that the macroporous hydrogel indeed has high permeability, which is critical to free transport of nutrients and wastes for normal cell metabolism. In contrast, a hydrogel without macroporous structures is insufficient for fast molecular transport, since previous work has shown that molecular transport for cell support in a normal non-macroporous hydrogel is ususally limited to $150-200 \mu \mathrm{m} .{ }^{49}$

The VEGF release results showed that approximately $89.6 \%$ and $94.7 \%$ of VEGF was released from the macroporous Apt (-) hydrogel in the M200 medium by day 1 and day 2, respectively (Figure 3C). By contrast, approximately $4.2 \%$ and $7.7 \%$ of VEGF was released from the macroporous Apt (+) hydrogel by day 1 and day 2, respectively. These data clearly demonstrate that the initial burst VEGF release was significantly reduced in the presence of the aptamer. Moreover, VEGF was released from the macroporous Apt (+) hydrogel in a sustained manner afterwards. The cumulative VEGF release was 20.3 and $28.1 \%$ by days 7 and 14, respectively (Figure 3C). Since in vitro cell cutlure and in vivo applications both involve serum that may influence the release kinetics, we further studied the capability of the aptamer in controlling VEGF release by incubating the macroporous Apt (+) hydrogels in three different release media. The results showed that VEGF was released with the same trend. The rate of VEGF release in LSGS-supplemented M200 was slightly higher than that in DPBS and M200. Since LSGS-supplemented M200 contains serum, it suggests that the chemically modified RNA aptamer had sufficient stability in prolonging VEGF release in biological fluids (Figure 3D). Thus, the aptamer-mediated release of soluble growth factors would be useful as biochemical cues to support cell metabolism.

Macroporous materials have been studied for controlled release of signaling molecules. The typical example is collagen sponge that has been approved by Food and Drug Administration (FDA) for controlled release of bone morphogenetic proteins in the clinic. ${ }^{53}$ However, the results showed that more than $50 \%$ and $90 \%$ of protein drugs were released from collagen sponge by days 7 and 14 , respectively. ${ }^{54}$ Presumably because of this fast release profile, to induce therapeutic effects, a very high amount of protein drugs were loaded into collagen sponge for implantation. ${ }^{55}$ Clinical results have indicated that the use of a large amount of protein drugs and the fast release could cause severe side effects and even increase cancer risk. ${ }^{56}$ In contrast, our data clearly show that a highly permeable 
macroporous hydrogel could strongly sequester soluble signaling molecules and enable their sustained release when chemically conjugated with oligonucleotide aptamers.

\subsection{Examination of VEGF bioactivity}

Two experiments were performed to evaluate and compare the potential of the Apt (-) and Apt (+) hydrogels in supporting normal cell metabolism using the tube formation assay. In the first experiment, the daily release media without any dilution were directly used for treatment of HUVECs. In the second experiment, VEGF remaining in the hydrogels was extracted and diluted to the same concentration as the stock solution.

The results showed that the release media collected at day 7 and day 14 in the Apt (-) group could not stimulate cell alignment and tube formation (Figure 4A), suggesting that the VEGF bioactivity was low in these release media. However, those collected at day 7 and day 14 in the Apt (+) group stimulated $73 \%$ and $43 \%$ of the cells to self-assemble and form tubes with the length of 1,088 and $550 \mu \mathrm{m} / \mathrm{mm}^{2}$, respectively (Figure 4A). This difference between the Apt (-) and Apt (+) groups is consistent with the VEGF release data showing that more than $95 \%$ of VEGF was released from the Apt (-) hydrogel during the first two days with little amount left for release during the following days whereas VEGF was released from the Apt (+) hydrogel in a sustained manner (Figure 3C). These data further suggest that the aptamer plays an important role of prolonging the release of soluble signaling molecules to local microenvironment of the macroporous hydrogel.

Since it is important for signaling molecules in a polymeric system to maintain high bioactivity during the release period, we further examined the bioactivity of VEGF retained inside the chimeric hydrogel. VEGF was extracted from hydrogels at day 7 and day 14 by inactivation of aptamer, and diluted to 10 and $20 \mathrm{ng} / \mathrm{mL}$, respectively, since these two concentrations are commonly used to evaluate the VEGF bioactivity in the tube formation assay. HUVECs treated with the VEGF solutions were self-assembled to form tubes (Figure 4B and $4 \mathrm{C}$ ). When $10 \mathrm{ng} / \mathrm{mL}$ of VEGF was used to treat HUVECs, the percentages of HUVECs participating in the formation of tubes were $98.9 \%, 96.7 \%$, and $83.7 \%$ in the stock media, triggered media at day 7 and triggered media at day 14 groups, respectively (Figure 4B). These differences are not statistically significant. The total lengths of the tubes in these three groups were 4,693, 4,027 and 2,389 $\mu \mathrm{m} / \mathrm{mm}^{2}$, respectively (Figure 4B). The lengths of the tubes induced by the stock VEGF solution and the VEGF extracted from the hydrogel at day 14 were statistically different (Figure 4B). When $20 \mathrm{ng} / \mathrm{mL}$ of VEGF was used to treat HUVECs, there was no statistically significant difference among these three VEGF samples in either cell participation in the tube formation or the total tube length (Figure 4B).

VEGF is an important growth factor to activate VEGF receptors for regulation of PI3K and FAK that play an important role of promoting endothelial cells survival and angiogenesis. ${ }^{57,58}$ A higher level of VEGF bioactivity leads to the formation of longer tubes. Thus, the results suggest that VEGF inside the macroporous hydrogel could maintain 50\% bioactivity in comparison to the stock VEGF solution by day 14. Proteins are not stable. Many factors can lead to protein degradation or denaturation, including protein fragmentation, aspartate isomerization, protein oxidation, deamidation, and disulfide bond shuffling. ${ }^{59}$ Therefore, it is challenging to maintain high bioactivity of therapeutic proteins 
(e.g., VEGF) for a long period of time. ${ }^{60,59}$ For instance, $\mathrm{Gu}$ and coworkers found that the bioactivity of VEGF in photocrosslinked elastomers decreased to $~ 15 \%$ after 10 days of in vitro release; ${ }^{61}$ Ekaputra and coworkers showed that the bioactivity of VEGF in a hydrogel composite decreased to $5 \%$ after 7 days of in vitro release. ${ }^{62}$ The difference between the previous studies and ours most likely results from the facts that the synthesis of the chimeric hydrogel was decoupled from VEGF loading and that the aptamers strongly bound VEGF in the chimeric hydrogel. Thus, our data demonstrate that the aptamer functionalized macroporous hydrogel can not only sequester growth factors for sustained release but also maintain their bioactivity.

\subsection{Evaluation of Cell Viability in the VEGF-loaded Macroprous Hydrogels}

After separately demonstrating the two functions of the chimeric hydrogel in loading cells and controlling growth factor release, we simultaneously loaded HUVECs and VEGF into the chimeric hydrogel. In each hydrogel, $6.25 \times 10^{5}$ cells and $200 \mathrm{ng}$ VEGF were loaded. Since cells often experience harsh microenvironment (e.g., lack of dynamic molecular transport) in real applications, the hydrogels were incubated in a serum-reduced medium for static cell culture. The cells in the hydrogels were evaluated after calcein AM staining.

At 12 hours, the cells in both Apt (-) and Apt (+) hydrogels exhibited a strong fluorescence intensity (Figure 5A and 5B), which indicates that the cells had a high activity in these hydrogels. However, the fluorescence intensity of the cells in the Apt (-) hydrogel quickly decreased with time (Figure 5A and 5B). It decreased by $70 \%$ from day 0.5 to day 3. In contrast, the fluorescence intensity of the cells in the Apt $(+)$ hydrogel virtually maintained the same during the first 3 days (Figure 5A and 5B). Moreover, except day 0.5, the fluorescence intensity of the cells in the Apt $(+)$ hydrogel was much stronger than that of the Apt (-) hydrogel (Figure 5A and 5B). On day 14, the fluorescence intensity of the Apt (+) hydrogel was $\sim 8$ times as much as that of the Apt (-) hydrogel.

Since VEGF can regualte PI3K and FAK pathways to enhance cell survival by activating VEGF receptors, ${ }^{57,58}$ it has been used in two different ways for functionalization of hydrogels ${ }^{63}$ One is to physically entrap soluble VEGF for sustained release; ${ }^{64}$ the other is to chemically conjugate VEGF as the cell binding sites. ${ }^{65}$ Both soluble and conjugated VEGF are able to support cell survival. ${ }^{64,65}$ In this work, VEGF was noncovalently entrapped in the hydrogel through physical binding to the anti-VEGF aptamer. It is important to note that this aptamer is a VEGF inhibitor, which has been shown in both preclinical and clinical studies. ${ }^{66,67}$ Since this aptamer is able to inhibit VEGF bioactivity, it was approved by FDA as an anti-angiogenic agent for treatment of age-related macular degeneration. ${ }^{67} \mathrm{In}$ another word, once VEGF binds to this aptamer, VEGF loses its bioactivity of binding to and stimulating VEGF receptors. ${ }^{66,67}$ Thus, the difference between the Apt (-) and Apt (+) hydrogels demonstrates that the Apt (+) hydrogel could release soluble VEGF in a sustained manner and the released VEGF could enhance the survival of HUVECs whereas the Apt (-) hydrogel could not.

The HUVECs in the macroporous hydrogel were further examined under the confocal microscope. Consistent with the macroscopic imaging analysis (Figure 5A), the microscopy images show that more living cells were observed in the Apt (+) hydrogel than in the Apt 
(-) hydrogel (Figure 5C). Some HUVECs in the Apt (+) hydrogels could connect together, which further indicate the viability of the HUVECs (Figure 5D). The effect of the macroporous structure on cell activity was also studied. The macroporous Apt (+) hydrogel was treated with the Geltrex solution that was gelated to block the macroporous structure (Figure 5E). The imaging data show that fewer cells survived in the pore (-) hydrogel than in the pore (+) hydrogel (Figure 5F). Since cell survival is important to the success of in vivo cell implantation, this difference clearly demonstrates that the macroporous structure is essential to maintain the activity of the cells in a hydrogel.

\section{CONCLUSIONS}

In summary, we synthesized a chimeric macroporous aptamer gelatin hydrogel using gelatin and an anti-VEGF oligonucleotide aptamer as a model. The macroporous structure of the chimeric hydrogel is highly permeable to molecular transport; gelatin provides HUVECs with binding sites for cell loading; and the aptamer strongly sequesters VEGF for sustained VEGF release with high bioactivity. Importantly, the cells can maintain higher activity in the VEGF-loaded macroporous hydrogel. Therefore, the chimeric macroporous aptamer-gelatin hydrogel holds potential to recapitulate the critical functions of the ECM for loading cells and growth factors. Future work will be pursued to demonstrate in vitro development of large-scale engineered tissue and in vivo implantation of cells for regenerative medicine.

\section{Supplementary Material}

Refer to Web version on PubMed Central for supplementary material.

\section{Acknowledgments}

We thank the Huck Institute Microscopy Facilities (University Park, PA), Shihui Li and Xuechen Shi for technical support. Research reported in this work was supported in part by the U.S. NSF CAREER program under award number DMR-1332351, the National Heart, Lung, and Blood Institute of the NIH under award number R01HL122311 and the Penn State Start-Up Fund.

\section{References}

1. Vlodavsky I, Folkman J, Sullivan R, Fridman R, Ishai-Michaeli R, Sasse J, Klagsbrun M. Proc Natl Acad Sci. 1987; 84:2292-2296. [PubMed: 3470794]

2. Robinson J, Gospodarowicz D. J Biol Chem. 1984; 259:3818-3824. [PubMed: 6706981]

3. Horacek MJ, Thompson JC, Dada MO, Terracio L. Acta Anat. 1993; 147:69-74. [PubMed: 8379294]

4. Martino MM, Mochizuki M, Rothenfluh DA, Rempel SA, Hubbell JA, Barker TH. Biomaterials. 2009; 30:1089-1097. [PubMed: 19027948]

5. Han SW, Roman J. Oncogene. 2006; 25:4341-4349. [PubMed: 16518410]

6. Huang W, Chiquet-Ehrismann R, Moyano JV, Garcia-Pardo A, Orend G. Cancer Res. 2001; 61:8586-8594. [PubMed: 11731446]

7. Eisenmann KM, McCarthy JB, Simpson MA, Keely PJ, Guan JL, Tachibana K, Lim L, Manser E, Furcht LT, Iida J. Nat Cell Biol. 1999; 1:507-513. [PubMed: 10587647]

8. Chevalier R. J Am Soc Nephrol. 1996; 7:1098-1105. [PubMed: 8866400]

9. Sieg DJ, Hauck CR, Ilic D, Klingbeil CK, Schaefer E, Damsky CH, Schlaepfer DD. Nat Cell Biol. 2000; 2:249-256. [PubMed: 10806474]

10. Furth ME, Atala A, Van Dyke ME. Biomaterials. 2007; 28:5068-5073. [PubMed: 17706763] 
11. Engel BJ, Constantinou PE, Sablatura LK, Doty NJ, Carson DD, Farach-Carson MC, Harrington DA, Zarembinski TI. Adv Healthc Mater. 2015; 4:1664-1674. [PubMed: 26059746]

12. Fong ELS, Martinez M, Yang J, Mikos AG, Navone NM, Harrington DA, Farach-Carson MC. Mol Pharm. 2014; 11:2040-2050. [PubMed: 24779589]

13. Gulfam M, Lee JM, Chung BG. Biotechnol Prog. 27:466-472. [PubMed: 21344675]

14. Hwang YS, Cho J, Tay F, Heng JYY, Ho R, Kazarian SG, Williams DR, Boccaccini AR, Polak JM, Mantalaris A. Biomaterials. 2009; 30:499-507. [PubMed: 18977027]

15. Byrne ME, Park K, Peppas NA. Adv Drug Deliv Rev. 2002; 54:149-161. [PubMed: 11755710]

16. Ehrbar M, Schoenmakers R, Christen EH, Fussenegger M, Weber W. Nat Mater. 2008; 7:800-804. [PubMed: 18690239]

17. Shu XZ, Liu Y, Palumbo F, Prestwich GD. Biomaterials. 2003; 24:3825-3834. [PubMed: 12818555]

18. Kraehenbuehl TP, Zammaretti P, Van der Vlies AJ, Schoenmakers RG, Lutolf MP, Jaconi ME, Hubbell JA. Biomaterials. 2008; 29:2757-2766. [PubMed: 18396331]

19. Andreopoulos FM, Beckman EJ, Russell AJ. Biomaterials. 1998; 19:1343-1352. [PubMed: 9758034]

20. Jeon O, Bouhadir KH, Mansour JM, Alsberg E. Biomaterials. 2009; 30:2724-2734. [PubMed: 19201462]

21. Rowley JA, Madlambayan G, Mooney DJ. Biomaterials. 1999; 20:45-53. [PubMed: 9916770]

22. Seo SJ, Akaike T, Choi YJ, Shirakawa M, Kang IK, Cho CS. Biomaterials. 2005; 26:3607-3615. [PubMed: 15621251]

23. Andreopoulos FM, Persaud I. Biomaterials. 2006; 27:2468-2476. [PubMed: 16321436]

24. Nie T, Baldwin A, Yamaguchi N, Kiick KL. J Control Release. 2007; 122:287-296. [PubMed: 17582636]

25. Burdick JA, Mason MN, Hinman AD, Thorne K, Anseth KS. J Control Release. 2002; 83:53-63. [PubMed: 12220838]

26. Stachowiak AN, Bershteyn A, Tzatzalos E, Irvine DJ. Adv Mater. 2005; 17(4):399-403.

27. Hollister SJ. Nat Mater. 2005; 4:518-524. [PubMed: 16003400]

28. Ellington AD, Szostak JW. Nature. 1990; 346:818-822. [PubMed: 1697402]

29. Tuerk C, Gold L. Science. 1990; 249:505-510. [PubMed: 2200121]

30. Wei B, Cheng I, Luo KQ, Mi Y. Angew Chem Int Ed. 2008; 47:331-333.

31. Xiong X, Liu H, Zhao Z, Altman MB, Lopez-Colon D, Yang CJ, Chang LJ, Liu C, Tan W. Angew Chem Int Ed. 2013; 52:1472-1476.

32. Zhu Z, Guan Z, Jia S, Lei Z, Lin S, Zhang H, Ma Y, Tian ZQ, Yang CJ. Angew Chem Int Ed. 2014; 53:12503-12507.

33. Wu ZS, Zheng F, Shen GL, Yu RQ. Biomaterials. 2009; 30:2950-2955. [PubMed: 19254812]

34. Tong R, Yala L, Fan TM, Cheng J. Biomaterials. 2010; 31:3043-3053. [PubMed: 20122727]

35. Guo J, Gao X, Su L, Xia H, Gu G, Pang Z, Jiang X, Yao L, Chen J, Chen H. Biomaterials. 2011; 32:8010-8020. [PubMed: 21788069]

36. Zheng D, Seferos DS, Giljohann DA, Patel PC, Mirkin CA. Nano Lett. 2009; 9:3258-3261. [PubMed: 19645478]

37. Battig MR, Soontornworajit B, Wang Y. J Am Chem Soc. 2012; 134:12410-12413. [PubMed: 22816442]

38. Soontornworajit B, Zhou J, Shaw MT, Fan TH, Wang Y. Chem Commun. 2010; 46:1857-1859.

39. Battig MR, Huang Y, Chen N, Wang Y. Biomaterials. 2014; 35:8040-8048. [PubMed: 24954732]

40. Nichol JW, Koshy ST, Bae H, Hwang CM, Yamanlar S, Khademhosseini A. Biomaterials. 2010; 31:5536-5544. [PubMed: 20417964]

41. Balakrishnan B, Mohanty M, Umashankar PR, Jayakrishnan A. Biomaterials. 2005; 26:6335-6342. [PubMed: 15919113]

42. Liu Y, Chan-Park MB. Biomaterials. 2009; 30:196-207. [PubMed: 18922573]

43. Ruckman J, Green LS, Beeson J, Waugh S, Gillette WL, Henninger DD, Claesson-Welsh L, Janjić N. J Biol Chem. 1998; 273:20556-20567. [PubMed: 9685413] 
44. Udenfriend S, Stein S, Böhlen P, Dairman W, Leimgruber W, Weigele M. Science. 1972; 178:871-872. [PubMed: 5085985]

45. Chen J, Park K. J Control Release. 2000; 65:73-82. [PubMed: 10699272]

46. Li S, Chen N, Zhang Z, Wang Y. Biomaterials. 2013; 34:460-469. [PubMed: 23083933]

47. Ashikari-Hada S, Habuchi H, Kariya Y, Kimata K. J Biol Chem. 2005; 280:31508-31515. [PubMed: 16027124]

48. Hoffman AS. J Biomater Sci Polym Ed. 1999; 10:1011-1014. [PubMed: 10591129]

49. Annabi N, Nichol JW, Zhong X, Ji C, Koshy S, Khademhosseini A, Dehghani F. Tissue Eng Part B Rev. 2010; 16:371-383. [PubMed: 20121414]

50. Nuttelman CR, Rice MA, Rydholm AE, Salinas CN, Shah DN, Anseth KS. Prog Polym Sci. 2008; 33:167-179. [PubMed: 19461945]

51. Zweier JL, Kuppusamy P, Lutty GA. Proc Natl Acad Sci. 1988; 85:4046-4050. [PubMed: 2836868]

52. Ruckman J, Green LS, Beeson J, Waugh S, Gillette WL, Henninger DD, Claesson-Welsh L, Janjic N. J Biol Chem. 1998; 273:20556-20567. [PubMed: 9685413]

53. Geiger M, Li RH, Friess W. Adv Drug Deliv Rev. 2003; 55:1613-1629. [PubMed: 14623404]

54. Uludag H, Gao T, Porter TJ, Friess W, Wozney JM. J Bone Joint Surg Am. 2001; 83A(Suppl):S128-S135. [PubMed: 11314790]

55. Bouxsein ML, Turek TJ, Blake CA, D’Augusta D, Li X, Stevens M, Seeherman HJ, Wozney JM. J Bone Joint Surg Am. 2001; 83-A:1219-1230. [PubMed: 11507131]

56. Carragee EJ, Chu G, Rohatgi R, Hurwitz EL, Weiner BK, Yoon ST, Comer G, Kopjar B. J Bone Joint Surg Am. 2013; 95:1537-1545. [PubMed: 24005193]

57. Zachary I. Cardiovasc Res. 2001; 49:568-581. [PubMed: 11166270]

58. Gupta VK, Jaskowiak NT, Beckett MA, Mauceri HJ, Grunstein J, Johnson RS, Calvin DA, Nodzenski E, Pejovic M, Kufe DW, Posner MC, Weichselbaum RR. Cancer J. 8:47-54. [PubMed: 11895203]

59. Rathore N, Rajan RS. Biotechnol Prog. 24:504-514. [PubMed: 18484778]

60. Fu K, Klibanov AM, Langer R. Nat Biotechnol. 2000; 18:24-25. [PubMed: 10625383]

61. Gu F, Neufeld R, Amsden B. Eur J Pharm Biopharm. 2007; 66:21-27. [PubMed: 16996254]

62. Ekaputra AK, Prestwich GD, Cool SM, Hutmacher DW. Biomaterials. 2011; 32:8108-8117. [PubMed: 21807407]

63. Lee K, Silva EA, Mooney DJ. J R Soc Interface. 2011; 8:153-170. [PubMed: 20719768]

64. Tabata Y, Miyao M, Ozeki M, Ikada Y. J Biomater Sci Polym Ed. 2000; 11:915-930. [PubMed: 11211086]

65. Leslie-Barbick JE, Moon JJ, West JL. J Biomater Sci Polym Ed. 2009; 20:1763-1779. [PubMed: 19723440]

66. Trujillo CA, Nery AA, Alves JM, Martins AH, Ulrich H. Clin Ophthalmol. 2007; 1:393-402. [PubMed: 19668516]

67. Ng EWM, Shima DT, Calias P, Cunningham ET, Guyer DR, Adamis AP. Nat Rev Drug Discov. 2006; 5:123-132. [PubMed: 16518379] 
A<smiles>C=C(C)C(=O)Nc1ccccc1</smiles>

B

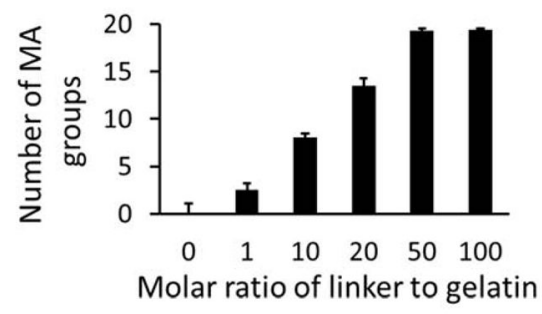

C

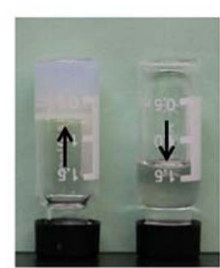

D
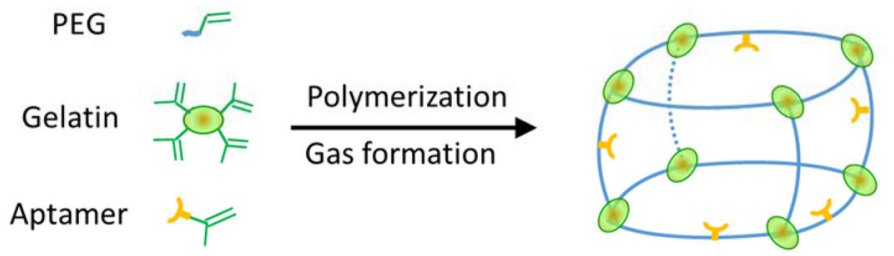

E

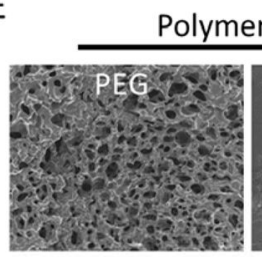

Polymerization + gas formation

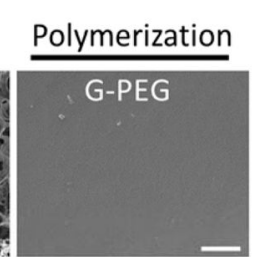

F

G-PEG

PEG

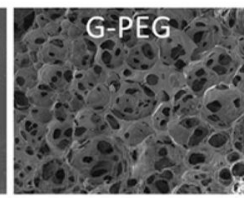

G

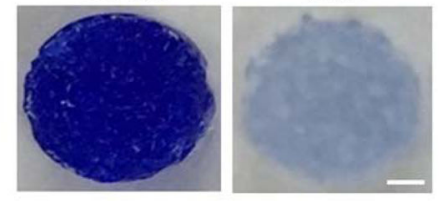
Apt (+) Apt (-)
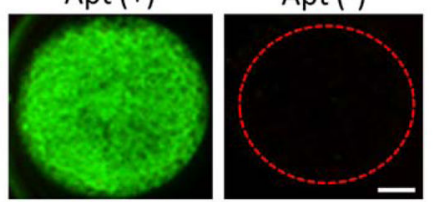

Figure 1.

Synthesis and characterization of hydrogels. A) Functionalization of gelatin with MA-NHS to synthesize gelatin-MA. B) Quantification of conjugation efficiency. Linker: MA-NHS. C) Formation of PEG hydrogels with the mixture of PEGMA and gelatin-MA or gelatin. With gelatin-MA, the pregel solution formed a hydrogel (left); with gelatin, the pregel solution did not form a hydrogel (right). D) Schematic illustration of synthesizing the chimeric macroporous hydrogel with free radical polymerization coupled with gas formation. E) SEM images of the hydrogels synthesized with two different reaction conditions. The first three hydrogels were synthesized with the coupled reactions of polymerization and gas formation. The fourth hydrogel was synthesized with only free radical polymerization. Pure PEGDA was used as control in forming PEG hydrogel. G-PEG indicates the gelatin-PEG hydrogel (the molar ratio of PEG and gelatin was 800:1). Scale bar: $500 \mu \mathrm{m}$. F) Examination of gelatin incorporation into the hydrogels with Coomassie Blue staining. Scale bars: $2 \mathrm{~mm}$. G) Examination of aptamer incorporation into the hydrogels with a FAM-labelled complementary oligonucleotide. Apt: aptamer; +/-: with/without. Scale bars: $2 \mathrm{~mm}$. 
A

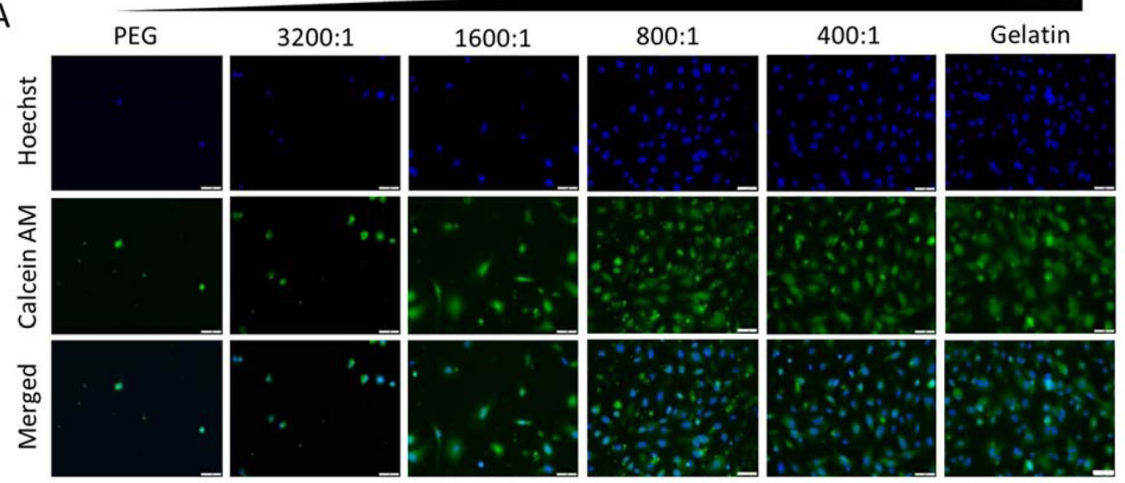

B

C
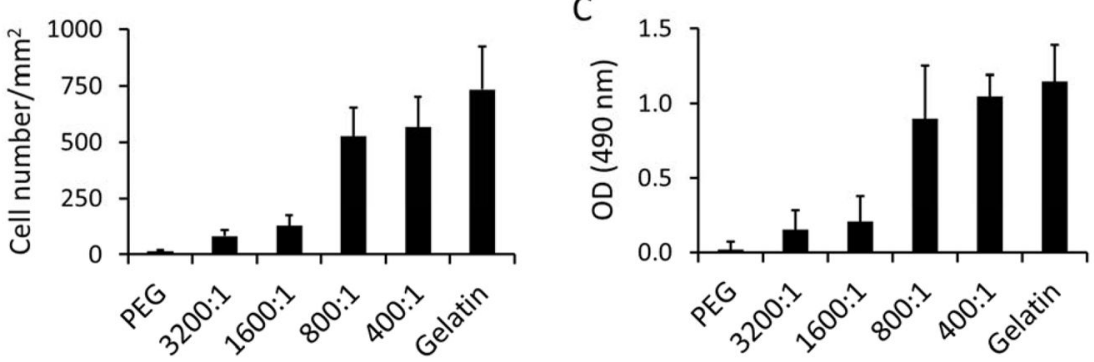

D

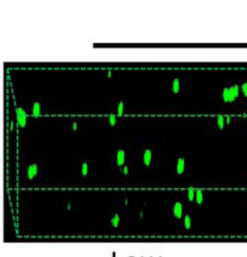

G-PEG

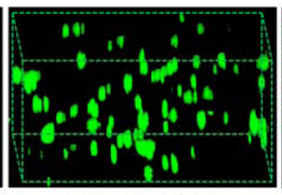

Intermediate

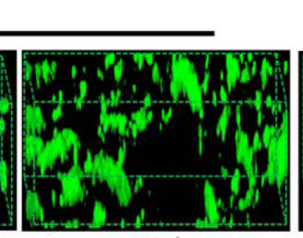

High

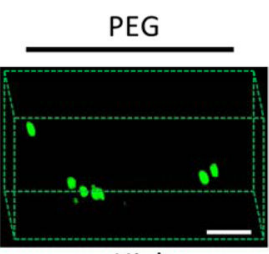

High

Figure 2.

Examination of cell binding and cell loading. A) Micrographs of cell adhesion. The HUVEC suspension of $250 \mu \mathrm{L}$ with $2.5 \times 10^{4}$ cells was seeded on the hydrogel. After overnight incubation, the cells were stained with Hoechst 33342 and calcein AM. The numbers indicate the molar ratios of PEGMA to gelatin-MA. The pure PEG hydrogel was synthesized with PEGDA. Scale bar: $100 \mu \mathrm{m}$. B) Quantification of HUVECs on the hydrogels using Image J. C) Examination of HUVECs on the hydrogels using the MTS assay. D) Confocal microscopy images of the HUVEC-loaded PEG hydrogels with and without gelatin. Low: $2.5 \times 10^{4}$ cells; intermediate: $1.25 \times 10^{5}$ cells; high: $6.25 \times 10^{5}$ cells. These numbers mean the initial cell loading amounts. Before the confocal microscopy imaging, hydrogels were treated with calcein AM for cell staining and washed to remove unbound cells. Scale bar: $100 \mu \mathrm{m}$. PEGDA was used as control in forming pure PEG hydrogel. G-PEG indicates the gelatin-PEG hydrogel (the molar ratio of PEG and gelatin was 800:1). 
A

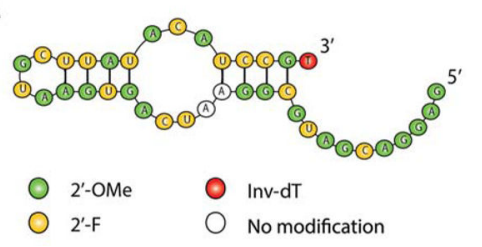

C

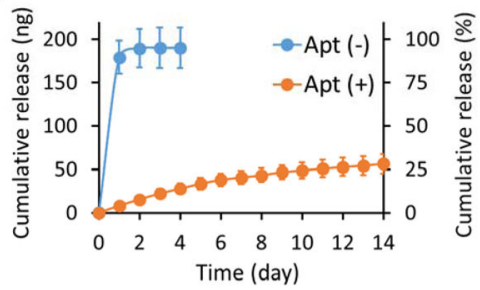

B
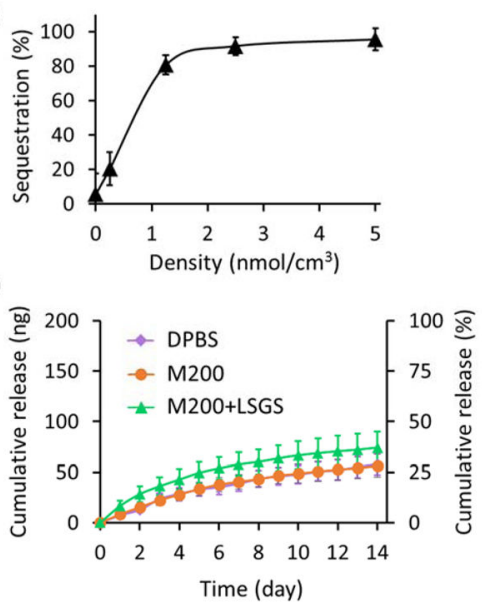

Figure 3.

Examination of VEGF sequestration and release. A) Composition and structure of the antiVEGF RNA aptamer. The secondary structure was generated by using the RNAstructure software (Version 5.3, from Mathews Lab). 2'-OMe: 2'O-methyl modification; 2'-F: 2' fluorine modification; Inv-dT: $3^{\prime}$ inverted dT. B) VEGF sequestration. $50 \mu \mathrm{L}$ of the solution with $200 \mathrm{ng}$ VEGF was added to each hydrogel and the samples were incubated at $4{ }^{\circ} \mathrm{C}$ for 24 hours. The sequestration efficiency was determined by the ratio of the amount VEGF remaining in the hydrogel after 24 hour release to that originally loaded into the hydrogel. C) Comparison of VEGF release from the hydrogels with (+) and without (-) the aptamer in the M200 medium. D) Comparison of VEGF release from the Apt (+) hydrogels in three different media including DPBS, M200, and LSGS-supplemented M200. The density of the aptamer was $2.5 \mathrm{nmol} / \mathrm{cm}^{3}$; the initial VEGF loading amount was $200 \mathrm{ng}$. In both C and D experiments, the hydrogels were directly incubated in the release media without any prewashing treatment. 
A

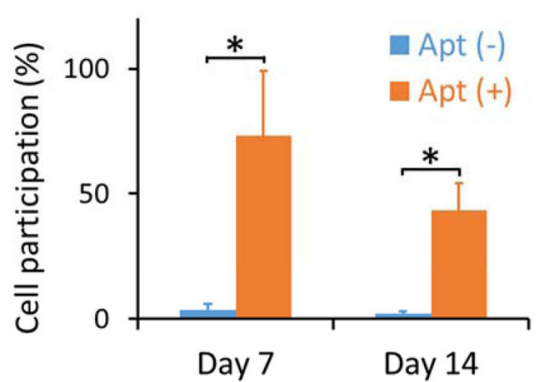

B

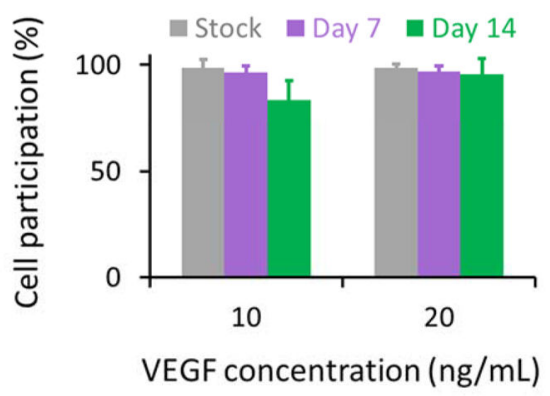

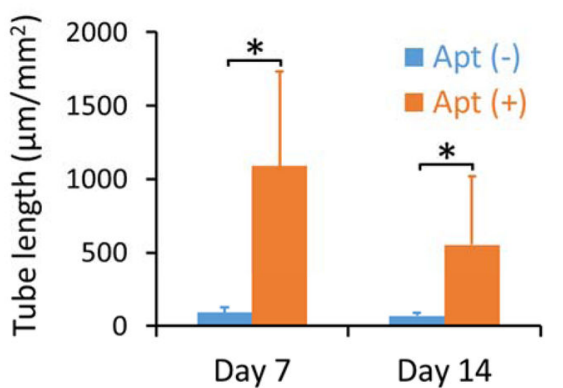

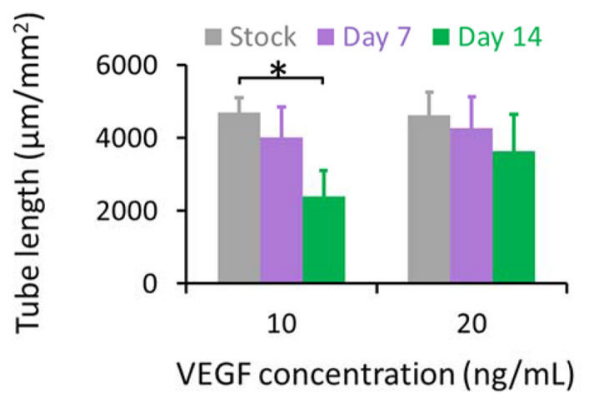

C

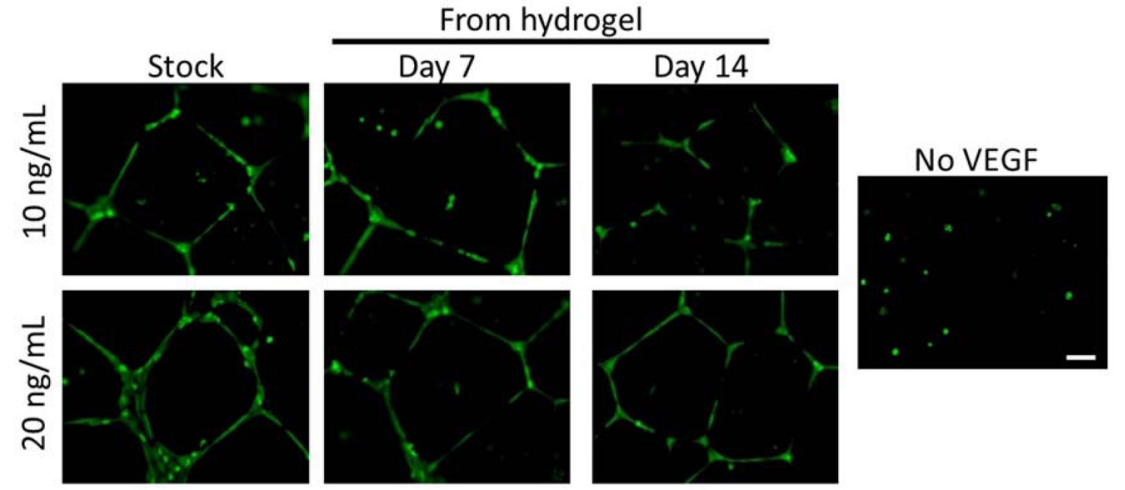

Figure 4.

Examination of VEGF bioactivity. A) Comparison of the VEGF release media collected at day 7 and day 14 in stimulating the tube formation. The release media from the Apt (+) and Apt (-) hydrogels were collected and directly used (without any dilution) to treat HUVECs. B) Comparison of the bioactivity between the stock VEGF solution and the VEGF freshly extracted from the hydrogels. The extracted VEGF samples were diluted to the same concentrations as the stock solutions. C) Representative images of cell tubes formed under different culture conditions. Scale bar: $100 \mu \mathrm{m}$. The cells were stained with calcein AM and imaged under the fluorescence microscope. Both cell participation into the tube formation and the total length of tubes were quantified. $* \mathrm{P}<0.05$. 
A

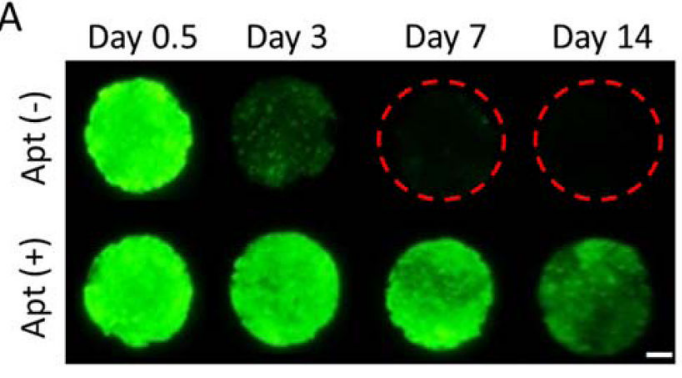

C

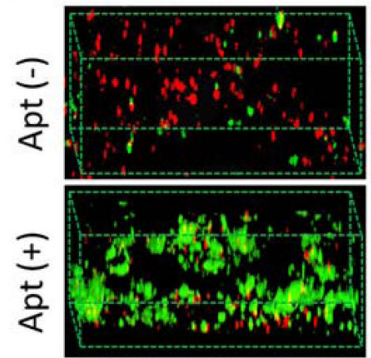

Day 7

E

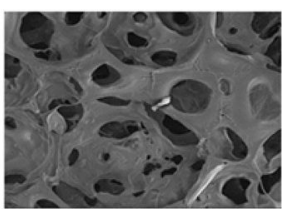

Pore (+)

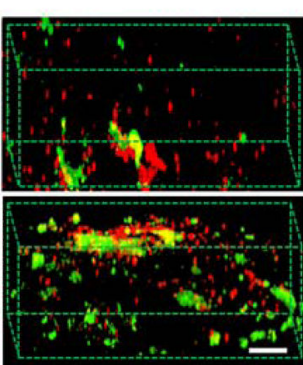

Day 14

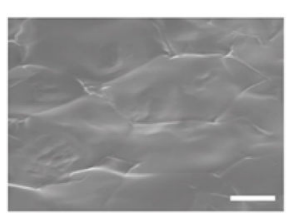

Pore (-)

B

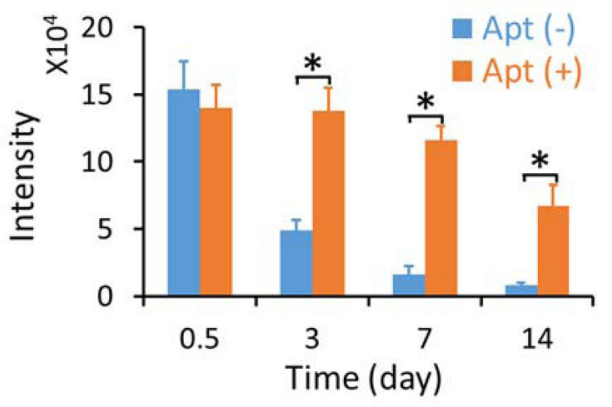

D

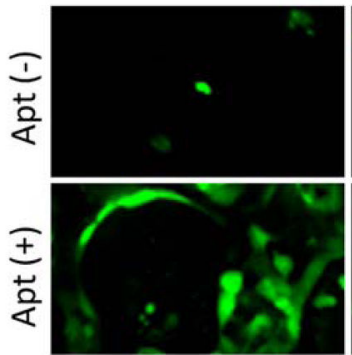

Day 7

F

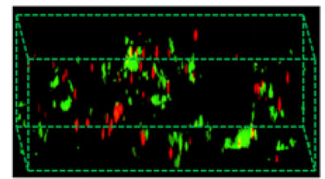

Day 7

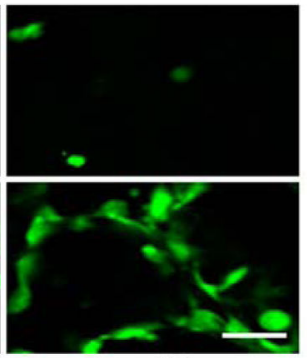

Day 14

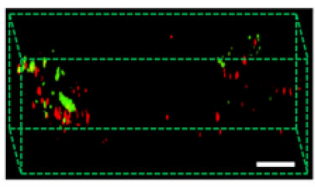

Day 14

Figure 5.

Evaluation of the chimeric hydrogel loaded with VEGF and HUVECs cultured at a static serum-reduced condition. A) Representative fluorescence images of the macroporous hydrogels. The cells were stained with calcein AM and the hydrogels were imaged using the Maestro Imaging System. Scale bar: $2 \mathrm{~mm}$. B) Quantification of cell fluorescence intensity of the macroporous hydrogels. $* \mathrm{P}<0.05$. C) Confocal microscopy images of live and dead cells in the macroporous hydrogels. Calcein AM and ethidium homodimer-1 were used to stain live (green) and dead (red) cells, respectively. Dead cells were often washed away during the sample treatment. Scale bars: $200 \mu \mathrm{m}$. D) Examination of cell-cell connection in the macroporous hydrogels. The images show that the surviving cells in the Apt (+) hydrogels could connect together to form tube-like structures. Scale bar: $30 \mu \mathrm{m}$. E) SEM images of the hydrogels. Pore (+): macroporous hydrogels without Geltrex treatment. Pore $(-)$ : macroporous hydrogels with Geltrex treatment. Scale bar: $100 \mu \mathrm{m}$. F) Confocal microscopy images of live and dead cells in the pore (-) hydrogels. Scale bar: $200 \mu \mathrm{m}$. 